\title{
Incidence and outcomes of patients with COVID-19 associated pulmonary aspergillosis (CAPA) in intensive care units: a systematic review and meta-analysis of 31 cohort studies
}

\author{
Wensen Chen ${ }^{1,2 \#}$, Cheng Yin ${ }^{3 \#}$, Ming Zhong ${ }^{4}$, Bijie Hu${ }^{5}$, Xiaodong Gao ${ }^{6}$, Kai Zhang ${ }^{7}$, Yun Liu ${ }^{8}$, \\ Guihua Zhuang ${ }^{1}$
}

${ }^{1}$ Department of Epidemiology and Biostatistics, School of Public Health, Xi'an Jiaotong University Health Science Center, Xi'an, China; ${ }^{2}$ Office of Infection Management, The First Affiliated Hospital of Nanjing Medical University, Nanjing, China; ${ }^{3}$ Department of Clinical Laboratory, Jiangning Hospital Affiliated to Nanjing Medical University, Nanjing, China; ${ }^{4}$ Department of Critical Care Medicine, Zhongshan Hospital Fudan University, Shanghai, China; ${ }^{5}$ Department of Infectious Diseases, Zhongshan Hospital Fudan University, Shanghai, China; ${ }^{6}$ Department of Infection Management, Zhongshan Hospital Fudan University, Shanghai, China; ${ }^{7}$ Pancreas Center, The First Affiliated Hospital of Nanjing Medical University, Nanjing, China; ${ }^{8}$ School of Biomedical Engineering and Informatics, Nanjing Medical University, Nanjing, China

Contributions: (I) Conception and design: W Chen, B Hu, Y Liu, G Zhuang; (II) Administrative support: Y Liu, G Zhuang; (III) Provision of study materials or patients: W Chen, C Yin; (IV) Collection and assembly of data: W Chen, C Yin, M Zhong, X Gao; (V) Data analysis and interpretation: W Chen, K Zhang; (VI) Manuscript writing: All authors; (VII) Final approval of manuscript: All authors.

\#These authors contributed equally to this work.

Correspondence to: Guihua Zhuang. Department of Epidemiology and Biostatistics, School of Public Health, Xi'an Jiaotong University Health Science Center, Xi'an 710061, China. Email: zhuanggh@mail.xjtu.edu.cn; Yun Liu. School of Biomedical Engineering and Informatics, Nanjing Medical University, Nanjing 210096, China. Email: liuyun@njmu.edu.cn.

Background: We aimed to identify studies systematically that describe the incidence and outcome of COVID-19-related pulmonary aspergillosis (CAPA).

Methods: We searched ScienceDirect, PubMed, CNKI, and MEDLINE (OVID) from December 31, 2019 to November 20, 2021 for all eligible studies. Random-model was used to reported the incidence, allcause case fatality rate (CFR) and $95 \%$ confidence intervals (CIs). The meta-analysis was registered with PROSPERO (CRD42021242179).

Results: In all, thirty-one cohort studies were included in this study. A total of 3,441 patients with severe COVID-19 admitted to an intensive care unit (ICU) were investigated and 442 cases of CAPA were reported (30 studies). The pooled incidence rate of CAPA was 0.14 (95\% CI: $0.11-0.17, \mathrm{I}^{2}=0.0 \%$ ). Twenty-eight studies reported 287 deceased patients and 269 surviving patients. The pooled CFR of CAPA was $0.52(95 \%$ CI: $\left.0.47-0.56, I^{2}=3.9 \%\right)$. Interestingly, patients with COVID19 would develop CAPA at 7.28 days after mechanical ventilation (range, 5.48-9.08 days). No significant publication bias was detected in this metaanalysis.

Discussion: Patients with COVID-19 admitted to an ICU might develop CAPA and have high all-cause CFR. We recommend conducting prospective screening for CAPA among patients with severe COVID-19, especially for those who receive mechanical ventilation over 7 days.

Keywords: COVID-19-associated pulmonary aspergillosis (CAPA); incidence; case fatality rate (CFR)

Submitted Jul 25, 2021. Accepted for publication Jan 28, 2022.

doi: 10.21037/apm-21-2043

View this article at: https://dx.doi.org/10.21037/apm-21-2043 


\section{Introduction}

From December 2019, a pandemic of coronavirus disease 2019 (COVID-19) caused by the novel severe acute respiratory syndrome coronavirus 2 (SARS-CoV-2) spread rapidly from Asia to the rest of the world. About $5 \%$ of patients with COVID-19 require intensive care unit (ICU) management (1). Clinicians are alert to the possibility of bacterial or fungal infection and prescribe antibiotics to nearly $90 \%$ of antibiotic patients with COVID-19 in the ICU setting (2). Respiratory viruses (including SARSCoV-2) cause direct damage the airway epithelium, facilitating bacterial and fungi invasion. Furthermore, ciliary clearance is hampered by viral infection, resulting in immune dysfunction or dysregulation (3). With severe pulmonary inflammation, long-term exposure to broad-spectrum antibiotics, and receipt of immunosuppressive drugs, patients with severe COVID-19 are at risk for secondary infections (4). It was reported that $14 \%$ hospitalized patients with COVID-19 had bacterial co-infections in mixed ward or ICU settings (5). Increasing numbers of studies have drawn attention to the high prevalence of COVID-19-associated pulmonary aspergillosis (CAPA) in patients with severe COVID-19 (1,6-8) admitted to the ICU. A diagnosis of CAPA was made based on specific case definitions or diagnostic algorithms, including the AspICU algorithm, modified AspICU algorithm, influenzaassociated pulmonary aspergillosis (IAPA) criteria, the European Organization for Research and Treatment of Cancer/Invasive Fungal Infections Cooperative Group, the National Institute of Allergy and Infectious Diseases Mycoses Study Group (EORTC/MSG), the European Confederation for Medical Mycology, and the International Society for Human and Animal Mycology (ECMM/ ISHAM) consensus criteria. However, the exact incidence of CAPA remains unknown and opinions are polarized $(9,10)$. Currently, reports on the incidence and outcomes of CAPA remain inconclusive. To address this gap in the literature, we conducted this systematic review and meta-analysis of 31 cohort studies, with the aim of identifying studies that describe the incidence and outcome of CAPA. We present the article in accordance with the PRISMA reporting checklist (available at https://apm.amegroups.com/article/ view/10.21037/apm-21-2043/rc).

\section{Methods}

This systematic review was based on a prospectively registered protocol (PROSPERO CRD42021242179). The review process is illustrated in a flow diagram (Figure 1).

\section{Search strategy}

A search string was developed to identify original research studies reporting clinical characteristics and outcomes of patients with CAPA. The search was applied to the following databases: ScienceDirect, PubMed, CNKI, and MEDLINE (OVID). Searches were performed for each database from December 31, 2019 to November 20, 2021. The following key words were used in all database searches: "coronavirus", "COVID”, "COVID-19", "severe acute respiratory syndrome”, “coronavirus 2", "SARS-CoV-2", "Aspergillus", "pulmonary Aspergillus", "invasive Aspergillus", "invasive pulmonary Aspergillus", "COVID-19-Associated pulmonary Aspergillosis" and "intensive care unit".

\section{Study selection and eligibility criteria}

All references were collected and screened independently by two reviewers (W Chen and $\mathrm{K}$ Zhang) against a set of predefined eligibility criteria. Potentially eligible studies were selected for full-text analysis. Disagreements were resolved by consensus or by appeal to a third senior reviewer (Y Liu). All observational cohort studies reporting the incidence and outcome of CAPA among patients with COVID-19 admitted to the ICU were included in this meta-analysis. The exclusion criteria included case control studies, case reports, nonhuman research, and meeting abstracts without a full article.

\section{Quality assessment}

The quality of the included studies was assessed using the Newcastle-Ottawa Scale (NOS) by the two researchers who were involved in the literature search (W Chen and $\mathrm{K}$ Zhang), who assessed the quality of all included studies and discussed discrepancies until a consensus was reached with the corresponding author (G Zhuang). The study quality was divided into low quality ( 0 to 3 stars), moderate quality (4 to 6 stars), and high quality (7 to 9 stars).

\section{Data extraction and outcome measures}

Data were extracted for the following variables: study period, study design, location of study, definition of CAPA and outcome. The case fatality rate (CFR) was defined as 
Identification of studies via databases and registers

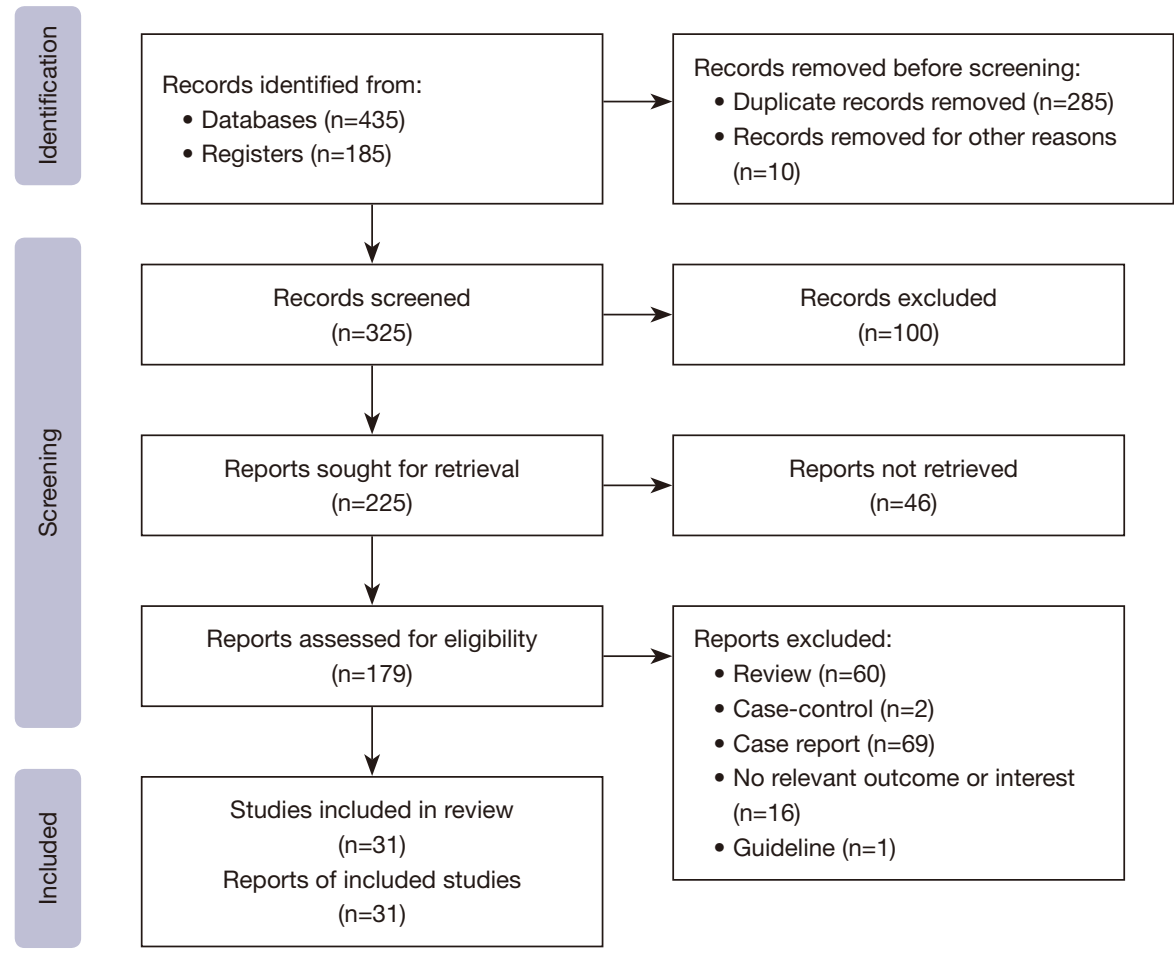

Figure 1 The flow diagram for this systematic reviews and meta-analyses.

the number of CAPA deaths divided by the total number of CAPA cases in the cohort. The primary outcome was allcause CFR during the ICU.

\section{Statistical analysis}

Random-effects meta-analysis was used to reported the incidence, all-cause CFR and with $95 \%$ confidence intervals (CIs) (11). Pooled proportions were computed using the inverse variance method and the variance-stabilizing Freeman-Tukey double arcsine transformation (12). Subgroup analysis was conducted based on the literature design (prospective or retrospective study), the type of research centre (single or multi-centre), and the clinical characteristics of the patients [e.g., acute respiratory distress syndrome (ARDS), mechanical ventilation]. The MantelHaenszel estimator was used to calculate the betweenstudy heterogeneity statistic Q, which was used in the DerSimonian-Laird estimator. The $\mathrm{I}^{2}$ statistic was used to present between-study heterogeneity, where $\mathrm{I}^{2}=50 \%$ was considered substantial. We assessed publication bias using Egger's test and by inspecting funnel plots. Statistical analyses were performed using the $\mathrm{R}$ software version 3.6.3 (R Foundation for Statistical Computing, 2016), with the package "meta" and Stata Special Edition 15.1 (Stata Corp., College Station, TX, USA). P values less than 0.05 were considered statistically significant.

\section{Results}

A total of 620 references were searched from MEDLINE, OVID, PubMed, and other sources. In all, we identified 31 observational cohort studies that were qualified according to our predefined inclusion and exclusion criteria (Figure 1).

\section{Characteristics of included studies}

Of the 31 studies, 9 (29.03\%) were from France, 4 (12.90\%) were from the Netherlands, 2 (6.45\%) were multi-national cohort study (1,6,10,13-20). Fourteen studies dealt with patients who received mechanical ventilation, eight studies were of patients with ARDS, one study was on 
Table 1 The incidence and CFR of CAPA in this meta-analysis

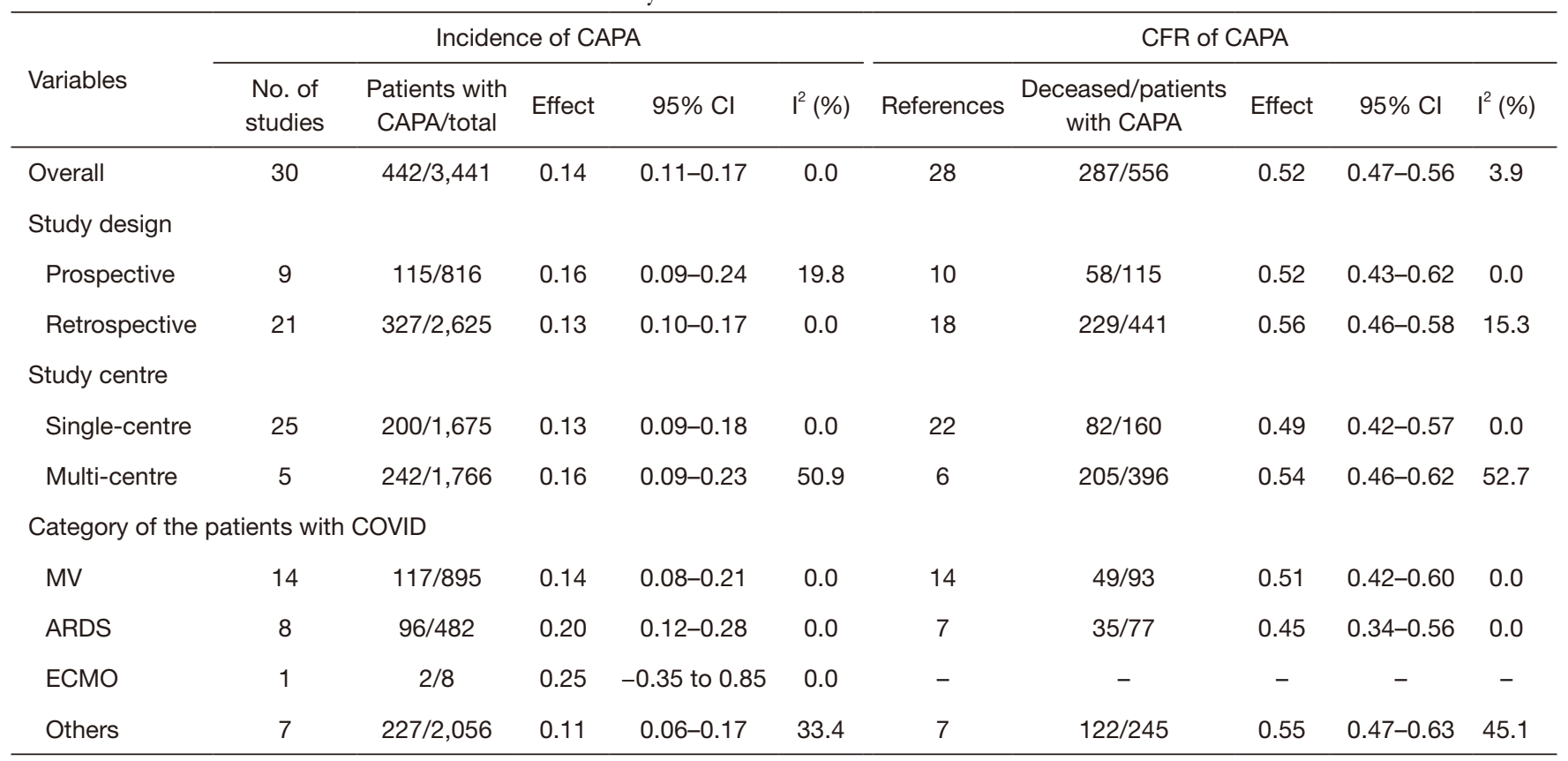

CFR, case fatality rate; CAPA, COVID-19-associated pulmonary aspergillosis; CI, confidence interval; MV, mechanical ventilation; ARDS, acute respiratory distress syndrome; ECMO, extracorporeal membrane oxygenation.

extracorporeal membrane oxygenation (ECMO), and seven studies included other patients with severe COVID-19 (21-40) (Table S1).

\section{Quality control of included studies}

The risk of bias included in the cohort studies was assessed using the NOS as presented in Table S2. A proportion of $93.55 \%(29 / 31)$ studies were over 7 stars, which is considered to high quality. All studies assessed the outcomes of interest based on medical record linkage (Table S3).

\section{Incidence of CAPA}

In the studies, 3,441 patients with severe COVID-19 admitted to an ICU were investigated and 442 cases of CAPA were reported. The incidence of CAPA was calculated as 0.14 (95\% CI: $\left.0.11-0.17, \mathrm{I}^{2}=0.0 \%\right)$. In the subgroup analysis by study design, the incidence of CAPA was calculated at 0.16 ( $95 \%$ CI: $\left.0.09-0.24, \mathrm{I}^{2}=19.8 \%\right)$ in the prospective studies and 0.13 (95\% CI: $0.10-0.17, \mathrm{I}^{2}=0.0 \%$ ) in the retrospective studies (Table 1). When stratified analysis was undertaken by research centre (multi-centre or single centre), the CAPA incidence was 0.13 (95\% CI: 0.09$\left.0.18, \mathrm{I}^{2}=0.0 \%\right)$ for the single centre studies and $0.16(95 \%$
CI: $\left.0.09-0.23, \mathrm{I}^{2}=50.9 \%\right)$ for the multi-centre studies. We found no significantly heterogeneity for calculation of the incidence of CAPA and significant publication bias according to Egger's regression test. Remarkably, we found that patients with COVID-19 admitted to an ICU would develop CAPA after approximately 7.28 days of mechanical ventilation (nine studies, 95\% CI: 5.48-9.08, $\mathrm{I}^{2}=46 \%$ ) (Figure 2).

\section{Colonisation by Aspergillus}

In five cohort studies including 365 patients with COVID-19 admitted to an ICU, 21 patients were assessed for Aspergillus colonisation. The calculated colonization rate of was $5.75 \%(21 / 365)$, suggesting that clinicians should comprehensively analyse Aspergillus colonisation combined with other clinical evidence (such as imaging and inflammatory factors, etc.) to judge whether the patient might develop CAPA and require further anti-fungal treatment.

\section{CFR of CAPA}

Twenty-eight studies were eligible for further analysis of allcause CFR with 287 deceased patients and 269 surviving patients. We found that the pooled CFR was 0.52 (95\% CI: 

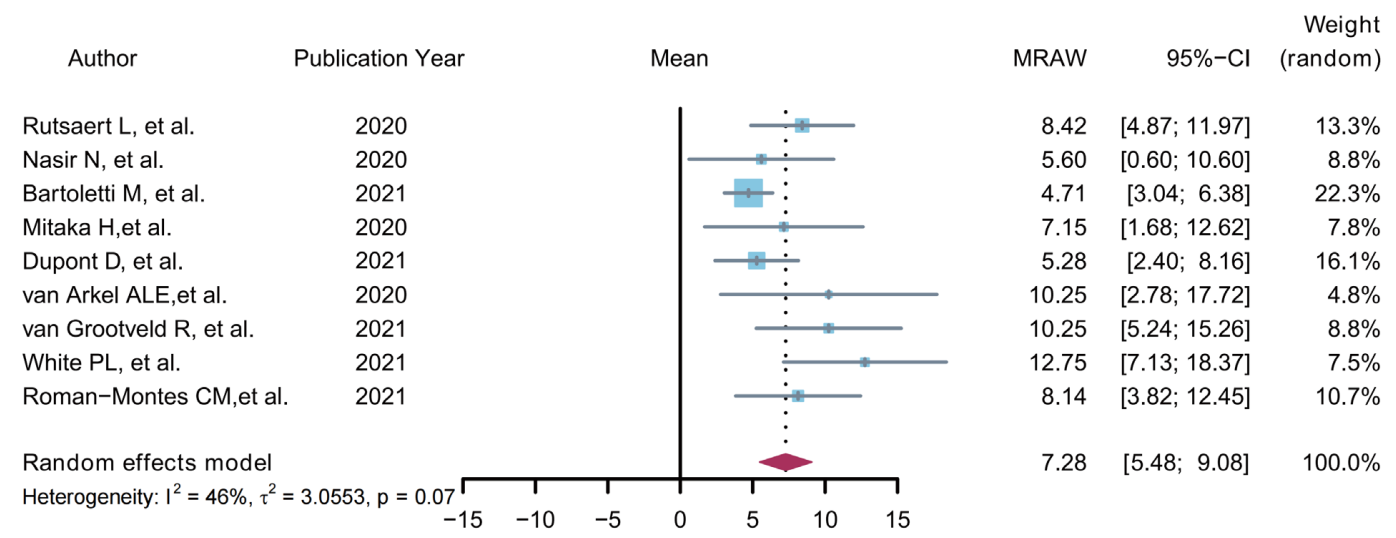

Figure 2 Time window of the patients developed CAPA. CAPA, COVID-19-associated pulmonary aspergillosis; CI, confidence interval; MRAW, meta-analysis of single means to calculate an overall mean using untransformed means (Raw).

$\left.0.47-0.56, \mathrm{I}^{2}=3.9 \%\right)$. In the subgroup analysis by study design, the calculated CFRs were 0.52 (95\% CI: $0.43-0.62, I^{2}=0.0$ ) for the prospective studies, 0.56 (95\% CI: $\left.0.46-0.58, \mathrm{I}^{2}=15.3 \%\right)$ for the retrospective studies, 0.49 (95\% CI: $0.42-0.57$, $\mathrm{I}^{2}=0.0 \%$ ) for the single centre studies, and 0.54 (95\% CI: 0.46 $0.62, \mathrm{I}^{2}=52.7 \%$ ) for the multi-centre studies (Table 1).

\section{Discussion}

We conducted this systematic review of 31 cohort studies and observed that $14 \%$ of patients with COVID-19 admitted to the ICU developed CAPA. Meanwhile, the median time to develop CAPA among patients with COVID-19 who received mechanical ventilation was 7.28 days. Studies reported that the incidence of CAPA varied from $1.9 \%$ to $27.7 \%(6,34,40)$. This wide divergence might result an underestimate of the risk of CAPA. Invasive pulmonary aspergillosis is a secondary infection of severe respiratory viral infection $(41,42)$ and these infections have been related with significant morbidity and mortality, even when appropriately diagnosed and treated. CAPA not only occurred in immunosuppressed patients (e.g., those with acute myeloid leukaemia), but also in immunocompetent patients with COVID-19 $(43,44)$. COVID-19 was independently associated with CAPA (31). We recommend that clinicians should be alert to the occurrence of CAPA in patients with COVID-19, especially in those receiving mechanical ventilation more than 7 days.

The occurrence of CAPA would exacerbate the deterioration of respiratory function, thus increasing the risk of death. We pooled 28 cohort studies and found that the all-cause CFR of patients with CAPA was $52 \%$. Among all included studies, a very high proportion of patients with COVID-19 received mechanical ventilation. The proportion of patients receiving baseline mechanical ventilation and 14 studies were $100 \%$ mechanical ventilation. A systematic review has revealed that the CFR of patients with COVID-19 receiving mechanical ventilation was $45 \%$ (95\% CI: $39-52 \%$ ) (45). Meijer and others conducted a small-sample cohort study (66 ICU patients with COVID-19) and found that the incidence of CAPA was $19.7 \%(\mathrm{n}=13 / 66)$, and the fatality rate of a single study was $46.2 \%(6 / 13)$; the author's team listed some of the articles that have been published and analyzed for the CAPA fatality rate. The pooled CFR for CAPA was $47 \%(62 / 132)(25)$.

The NOS was used to evaluate the quality of the included articles. We found that the selected studies were of high quality. However, we observed significant heterogeneity in the pooled analysis of the incidence and CFR of CAPA in the subgroup analysis with multi-centre. Many hospitals do not commonly perform bronchoscopy for CAPA diagnosis or use PCR tests for Aspergillus species (46). Non-standardized definitions of CAPA, differences in detection methods (47), and variations among research designs might have contributed to heterogeneity across the studies, which requires further investigation.

Meanwhile, we have some limitation in this study. The included literature did not provide the clear profile of the patients with COVID-19 developed with CAPA. In this case, we only pooled the time window for CAPA in mechanically ventilated patients. On the other hand, there was lack of a unified definition of CAPA and a standard 
diagnostic algorithm in the published studies. Although we strictly controlled quality based on the study entry and exclusion criteria, heterogeneity between studies was still unavoidable. We subsequently conducted a subgroup analysis according to different definition and found no significant heterogeneity. Last but not the least, this metaanalysis did not explore the risk factors for mortality in patients with CAPA because of insufficient literature support. Further well-designed research and demonstration would be warranted in the future.

\section{Conclusions}

In this study, the pooled incidence of CAPA was estimated as $14 \%$ among patients with COVID-19 admitted to the ICU, with high rate of all cause-mortality and CFR. We recommend that clinicians establish standardized screening methods for CAPA and to identify high risk patients early, especially for patients who have been mechanically ventilated for more than 7 days.

\section{Acknowledgments}

We are grateful to all health-care workers of Critical Care Medical Team of Jiangsu Province for their fight against the COVID-19 in Wuhan (Yun Liu, the team leader) and their assistance for this meta-analysis.

Funding: This research was supported by the National Key R\&D Program of the Ministry of Science and Technology of the People's Republic of China (Grant No. 2020YFC0848100), the industry prospecting and common key technology key projects of Jiangsu Province Science and Technology Department (Grant No. BE2020721) and and the Clinical Capability Improvement Project of Jiangsu Province Hospital (Grant No. FSPH-MB-2020-10).

\section{Footnote}

Reporting Checklist: The authors have completed the PRISMA reporting checklist. Available at https://apm. amegroups.com/article/view/10.21037/apm-21-2043/rc

Conflicts of Interest: All authors have completed the ICMJE uniform disclosure form (available at https://apm. amegroups.com/article/view/10.21037/apm-21-2043/coif). The authors have no conflicts of interest to declare.

Ethical Statement: The authors are accountable for all aspects of the work in ensuring that questions related to the accuracy or integrity of any part of the work are appropriately investigated and resolved.

Open Access Statement: This is an Open Access article distributed in accordance with the Creative Commons Attribution-NonCommercial-NoDerivs 4.0 International License (CC BY-NC-ND 4.0), which permits the noncommercial replication and distribution of the article with the strict proviso that no changes or edits are made and the original work is properly cited (including links to both the formal publication through the relevant DOI and the license). See: https://creativecommons.org/licenses/by-nc-nd/4.0/.

\section{References}

1. Alanio A, Dellière S, Fodil S, et al. Prevalence of putative invasive pulmonary aspergillosis in critically ill patients with COVID-19. Lancet Respir Med 2020;8:e48-9.

2. Zhou F, Yu T, Du R, et al. Clinical course and risk factors for mortality of adult inpatients with COVID-19 in Wuhan, China: a retrospective cohort study. Lancet 2020;395:1054-62.

3. Herold S, Becker C, Ridge KM, et al. Influenza virusinduced lung injury: pathogenesis and implications for treatment. Eur Respir J 2015;45:1463-78.

4. Lescure FX, Bouadma L, Nguyen D, et al. Clinical and virological data of the first cases of COVID-19 in Europe: a case series. Lancet Infect Dis 2020;20:697-706.

5. Lansbury L, Lim B, Baskaran V, et al. Co-infections in people with COVID-19: a systematic review and metaanalysis. J Infect 2020;81:266-75.

6. Bartoletti M, Pascale R, Cricca M, et al. Epidemiology of Invasive Pulmonary Aspergillosis Among Intubated Patients With COVID-19: A Prospective Study. Clin Infect Dis 2021;73:e3606-14.

7. Machado M, Valerio M, Álvarez-Uría A, et al. Invasive pulmonary aspergillosis in the COVID-19 era: An expected new entity. Mycoses 2021;64:132-43.

8. Marr KA, Platt A, Tornheim JA, et al. Aspergillosis Complicating Severe Coronavirus Disease. Emerg Infect Dis 2021;27:18-25.

9. Salmanton-García J, Sprute R, Stemler J, et al. COVID19-Associated Pulmonary Aspergillosis, March-August 2020. Emerg Infect Dis 2021;27:1077-86.

10. Dellière S, Dudoignon E, Fodil S, et al. Risk factors associated with COVID-19-associated pulmonary aspergillosis in ICU patients: a French multicentric 
retrospective cohort. Clin Microbiol Infect 2020. [Epub ahead of print].

11. Hozo SP, Djulbegovic B, Hozo I. Estimating the mean and variance from the median, range, and the size of a sample. BMC Med Res Methodol 2005;5:13.

12. Nyaga VN, Arbyn M, Aerts M. Metaprop: a Stata command to perform meta-analysis of binomial data. Arch Public Health 2014;72:39.

13. Razazi K, Arrestier R, Haudebourg AF, et al. Risks of ventilator-associated pneumonia and invasive pulmonary aspergillosis in patients with viral acute respiratory distress syndrome related or not to Coronavirus 19 disease. Crit Care 2020;24:699.

14. Dupont D, Menotti J, Turc J, et al. Pulmonary aspergillosis in critically ill patients with Coronavirus Disease 2019 (COVID-19). Med Mycol 2021;59:110-4.

15. Chauvet P, Mallat J, Arumadura C, et al. Risk Factors for Invasive Pulmonary Aspergillosis in Critically Ill Patients With Coronavirus Disease 2019-Induced Acute Respiratory Distress Syndrome. Crit Care Explor 2020;2:e0244.

16. Gangneux JP, Reizine F, Guegan H, et al. Is the COVID-19 Pandemic a Good Time to Include Aspergillus Molecular Detection to Categorize Aspergillosis in ICU Patients? A Monocentric Experience. J Fungi (Basel) 2020;6:105.

17. Gouzien L, Cocherie T, Eloy O, et al. Invasive Aspergillosis associated with Covid-19: A word of caution. Infect Dis Now 2021;51:383-6.

18. Bretagne S, Sitbon K, Botterel F, et al. COVID-19Associated Pulmonary Aspergillosis, Fungemia, and Pneumocystosis in the Intensive Care Unit: a Retrospective Multicenter Observational Cohort during the First French Pandemic Wave. Microbiol Spectr 2021;9:e0113821.

19. Nasir N, Farooqi J, Mahmood SF, et al. COVID-19associated pulmonary aspergillosis (CAPA) in patients admitted with severe COVID-19 pneumonia: An observational study from Pakistan. Mycoses 2020;63:766-70.

20. Fekkar A, Lampros A, Mayaux J, et al. Occurrence of Invasive Pulmonary Fungal Infections in Patients with Severe COVID-19 Admitted to the ICU. Am J Respir Crit Care Med 2021;203:307-17.

21. Charalampous T, Alcolea-Medina A, Snell LB, et al. Evaluating the potential for respiratory metagenomics to improve treatment of secondary infection and detection of nosocomial transmission on expanded COVID-19 intensive care units. Genome Med 2021;13:182.

22. Borman AM, Palmer MD, Fraser M, et al. COVID-
19-Associated Invasive Aspergillosis: Data from the UK National Mycology Reference Laboratory. J Clin Microbiol 2020;59:e02136-20.

23. White PL, Dhillon R, Cordey A, et al. A National Strategy to Diagnose Coronavirus Disease 2019-Associated Invasive Fungal Disease in the Intensive Care Unit. Clin Infect Dis 2021;73:e1634-44

24. van Grootveld R, van Paassen J, de Boer MGJ, et al. Systematic screening for COVID-19 associated invasive aspergillosis in ICU patients by culture and PCR on tracheal aspirate. Mycoses 2021;64:641-50.

25. Meijer EFJ, Dofferhoff ASM, Hoiting O, et al. COVID19-associated pulmonary aspergillosis: a prospective singlecenter dual case series. Mycoses 2021;64:457-64.

26. Van Biesen S, Kwa D, Bosman RJ, et al. Detection of Invasive Pulmonary Aspergillosis in COVID-19 with Nondirected Bronchoalveolar Lavage. Am J Respir Crit Care Med 2020. [Epub ahead of print].

27. van Arkel ALE, Rijpstra TA, Belderbos HNA, et al. COVID-19-associated Pulmonary Aspergillosis. Am J Respir Crit Care Med 2020;202:132-5.

28. Janssen NAF, Nyga R, Vanderbeke L, et al. Multinational Observational Cohort Study of COVID-19-

Associated Pulmonary Aspergillosis1. Emerg Infect Dis 2021;27:2892-8.

29. Prattes J, Wauters J, Giacobbe DR, et al. Risk factors and outcome of pulmonary aspergillosis in critically ill coronavirus disease 2019 patients-a multinational observational study by the European Confederation of Medical Mycology. Clin Microbiol Infect 2021. [Epub ahead of print].

30. Koehler P, Cornely OA, Böttiger BW, et al. COVID-19 associated pulmonary aspergillosis. Mycoses 2020;63:528-34.

31. Lahmer T, Kriescher S, Herner A, et al. Invasive pulmonary aspergillosis in critically ill patients with severe COVID-19 pneumonia: Results from the prospective AspCOVID-19 study. PLoS One 2021;16:e0238825.

32. Nebreda-Mayoral T, Miguel-Gómez MA, March-Rosselló GA, et al. Bacterial/fungal infection in hospitalized patients with COVID-19 in a tertiary hospital in the Community of Castilla y León, Spain. Enferm Infecc Microbiol Clin (Engl Ed). 2020. [Epub ahead of print]. doi:10.1016/ j.eimc.2020.11.003.

33. Segrelles-Calvo G, Araújo GRS, Llopis-Pastor E, et al. Prevalence of opportunistic invasive aspergillosis in COVID-19 patients with severe pneumonia. Mycoses 2021;64:144-51.

34. Lamoth F, Glampedakis E, Boillat-Blanco N, et al. 
Incidence of invasive pulmonary aspergillosis among critically ill COVID-19 patients. Clin Microbiol Infect 2020;26:1706-8.

35. Buehler PK, Zinkernagel AS, Hofmaenner DA, et al. Bacterial pulmonary superinfections are associated with longer duration of ventilation in critically ill COVID-19 patients. Cell Rep Med 2021;2:100229.

36. Mitaka H, Perlman DC, Javaid W, et al. Putative invasive pulmonary aspergillosis in critically ill patients with COVID-19: An observational study from New York City. Mycoses 2020;63:1368-72.

37. Rutsaert L, Steinfort N, Van Hunsel T, et al. COVID-19associated invasive pulmonary aspergillosis. Ann Intensive Care 2020;10:71.

38. Wang J, Yang Q, Zhang P, et al. Clinical characteristics of invasive pulmonary aspergillosis in patients with COVID-19 in Zhejiang, China: a retrospective case series. Crit Care 2020;24:299.

39. Helleberg M, Steensen M, Arendrup MC. Invasive aspergillosis in patients with severe COVID-19 pneumonia. Clin Microbiol Infect 2021;27:147-8.

40. Roman-Montes CM, Martinez-Gamboa A, Diaz-Lomelí $\mathrm{P}$, et al. Accuracy of galactomannan testing on tracheal aspirates in COVID-19-associated pulmonary aspergillosis. Mycoses 2021;64:364-71.

41. Schwartz IS, Friedman DZP, Zapernick L, et al. High Rates of Influenza-Associated Invasive Pulmonary

Cite this article as: Chen W, Yin C, Zhong M, Hu B, Gao X, Zhang K, Liu Y, Zhuang G. Incidence and outcomes of patients with COVID-19 associated pulmonary aspergillosis (CAPA) in intensive care units: a systematic review and meta-analysis of 31 cohort studies. Ann Palliat Med 2022;11(7):2202-2209. doi: 10.21037/apm-21-2043
Aspergillosis May Not Be Universal: A Retrospective Cohort Study from Alberta, Canada. Clin Infect Dis 2020;71:1760-3.

42. Wauters J, Baar I, Meersseman P, et al. Invasive pulmonary aspergillosis is a frequent complication of critically ill H1N1 patients: a retrospective study. Intensive Care Med 2012;38:1761-8.

43. Hakamifard A, Hashemi M, Fakhim H, et al. Fatal disseminated aspergillosis in an immunocompetent patient with COVID-19 due to Aspergillus ochraceus. J Mycol Med 2021;31:101124.

44. Haglund A, Christensen S, Kristensen L, et al. Invasive pulmonary aspergillosis and hyperthermia in an immunocompetent patient with COVID-19. Med Mycol Case Rep 2021;31:29-31.

45. Lim ZJ, Subramaniam A, Ponnapa Reddy M, et al. Case Fatality Rates for Patients with COVID-19 Requiring Invasive Mechanical Ventilation. A Meta-analysis. Am J Respir Crit Care Med 2021;203:54-66.

46. Wahidi MM, Shojaee S, Lamb CR, et al. The Use of Bronchoscopy During the Coronavirus Disease 2019 Pandemic: CHEST/AABIP Guideline and Expert Panel Report. Chest 2020;158:1268-81.

47. Koehler P, Bassetti M, Chakrabarti A, et al. Defining and managing COVID-19-associated pulmonary aspergillosis: the $2020 \mathrm{ECMM} / \mathrm{ISHAM}$ consensus criteria for research and clinical guidance. Lancet Infect Dis 2021;21:e149-62. 